\title{
Viaggiando tra montagne, campagne e piccole isole: le scuole primarie rurali italiane motore per lo sviluppo della comunità
}

\section{Elisabetta Tomazzoli *}

\section{Riassunto}

L'obiettivo di questo lavoro è quello di astrarre le proprietà essenziali del rapporto tra le piccole scuole rurali e la comunità in cui sono inserite. Con i bambini al centro dell'attenzione, questa ricerca analizza l'interazione tra bambini, insegnanti, genitori, dirigenti e politici locali, residenti e famiglie straniere, così come il loro rapporto con l'ambiente naturale. Seguendo i fondamenti della metodologia Grounded Theory con un approccio costruttivista e Informed, sono state visitate 14 scuole primarie rurali italiane che includono almeno una pluriclasse. I risultati della ricerca evidenziano un elemento fondamentale che trascende l'eterogeneità delle realtà prese in esame e cioè la presenza di figure fortemente motivate (insegnanti, genitori, dirigenti, politici, ecc.) che vedono la scuola come motore per lo sviluppo della comunità. Tra queste figure, nell'analisi, una particolare attenzione è stata posta sugli insegnanti: è stato costruito il concetto di insegnante come intellettuale organico per definire l'insegnante che vive lui stesso la comunità, ma con l'occhio della ricerca storica, letteraria, naturale, musicale e con tutto l'ambiente.

\section{Parole chiave}

Relazione scuola-comunità, piccola scuola rurale, comunità rurale, pluriclasse, ruolo dell'insegnante, politiche educative.

Ricezione originale: 21 aprile 2020

Accettazione: 27 iuglio 2020

Pubblicazione: 16 de desembre de 2020

\section{Introduzione}

Perché occuparsi di piccole scuole rurali oggi? Dando uno sguardo generale, le seppur non numerose ricerche in questo campo ne documentano le esperienze educative e le prassi didattiche per argomentarne il valore, non solo all'interno del sistema scolastico in generale, ma anche per la comunità all'interno della quale queste piccole scuole sono inserite. Ad esempio, la ricerca sul territorio cileno portata avanti da Núñez-Muñoz, SolísAraya e Soto-Lago (2014) si intitola: ¿Qué sucede en las comunidades cuando se cierra la escuela rural? Un análisis psicosocial de la política de cierre de las escuelas rurales en Chile. La ricerca in ambito educativo ha comunque spesso trascurato queste piccole realtà, se non del tutto per quanto riguarda gli aspetti didattici nell'andare a vedere se le piccole e le grandi scuole preparano in maniera equa gli alunni, sicuramente per quanto riguarda la relazione tra piccole scuole e comunità. Nella revisione su 30 anni di ricerca sulle piccole scuole svedesi, la studiosa Aberg-Bengtsson conclude:

Sono stati trovati due focus principali: (i) la qualità dell'istruzione e i risultati scolastici degli alunni e (ii) gli aspetti economici della gestione delle scuole in diversi tipi di aree demografiche. Emerge un quadro concorde: (a) non vi sono indicazioni che le piccole scuole rurali non forniscano un'istruzione altrettanto buona rispetto ad altre scuole, ma (b) l'aumento della spesa per allievo e la diminuzione della popolazione nelle zone scarsamente popolate aumenta il rischio di chiusura delle scuole. L'importanza della

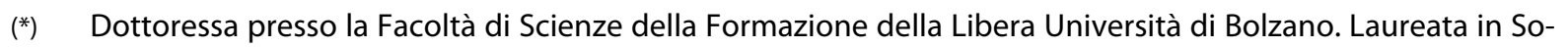
ciologia, i suoi studi in ambito educativo si occupano di scuole rurali pluriclasse e della loro relazione con la comunità all'interno della quale sono inserite. E-mail: etomazzolli@unibz.it 
piccola scuola rurale per la comunità riceve, nel migliore dei casi, una scarsa attenzione in questo genere di ricerche. (Aberg-Bengtsson, 2009, p. 100)

Simone White e Michael Corbett sottolineano «l'urgente necessità di spacchettare l'inesplorato predominio delle metodologie di ricerca prevalentemente metropolitane. Riteniamo che questo pregiudizio tenda a posizionare il rurale come un deficit, piuttosto che guardare ad esso come a qualcosa di diverso» (White, Corbett, a cura di, 2014, p. 1). Riassumendo, se da un lato vi è scarso interesse per la dimensione rurale all'interno della quale le piccole scuole sono inserite, dall'altra quando si prende in considerazione questo elemento lo si fa trattandolo come deficitario rispetto alla dimensione della città.

Un passo avanti riguardo all'attenzione posta sulle realtà scolastiche in Italia è stato fatto dall'organizzazione ministeriale INDIRE attraverso la stesura e la firma nel 2017 del Manifesto delle Piccole Scuole, finalizzato alla costruzione di una rete di piccole scuole italiane. Uno dei tre punti chiave del Manifesto (insieme all'esperienza della pluriclasse e alle tecnologie come strumento di inclusione), è la conservazione dei tratti distintivi propri delle piccole scuole dentro la comunità. Vi si legge: «ll loro rapporto con l'ambiente naturale, sociale e culturale può rappresentare una risorsa dalle forti potenzialità innovative nel momento in cui lega l'apprendimento alla realtà valorizzandola nel rispetto delle vocazioni territoriali» (INDIRE, http://www.indire.it/progetto/piccole-scuole/).

Anche la legge del 2015 nota con il nome di La buona scuola (LEGGE 13 luglio $2015 \mathrm{n}$. 107) ha previsto un documento allegato chiamato La buona scuola per le aree interne (http://www.istruzione.it/allegati/2015/labuonascuolaareeinterne.pdf), il quale prevede degli interventi strategici all'interno di aree-progetto individuate attraverso previe ricerche sul campo che hanno fatto emergere le criticità della scuola in questi territori. II contesto periferico di tali aree è messo sin da subito in relazione al fenomeno dello spopolamento e la scuola è vista come uno dei fattori che determinano la scelta di continuare a vivere $\mathrm{o}$ addirittura trasferirsi a vivere in queste zone:

La scuola rappresenta (con salute, mobilità e rete digitale) uno dei tre servizi di base da cui dipende la scelta di restare a vivere o di trasferirsi nelle aree interne del paese. Per tale ragione la Strategia nazionale per le aree interne lanciata dall'Italia finanziaria, attraverso la Legge di Stabilità, prevede la sperimentazione di interventi permanenti per migliorare la qualità della scuola. Le presenti linee guida, elaborate sulla base delle indicazioni contenute nella legge n. 107/2015, sulla «Riforma del sistema nazionale di istruzione e formazione», intendono delineare i possibili interventi da porre in essere nell'ambito della strategia di sviluppo delle aree interne. (La buona scuola per le aree interne, 2015, p. ii)

Infatti, la tendenza non solo in Italia ma un po' in tutta Europa è quella di chiudere le piccole scuole, creando centri più grandi in zone più popolate e meno periferiche. Vivo è di conseguenza in tutta Europa il dibattito sulla chiusura di queste realtà scolastiche e sul futuro dei piccoli centri rurali. Guardando al fenomeno attraverso la lente della pedagogia critica, al centro del discorso sulla chiusura delle piccole scuole vengono posti gli approcci politici, economici ed educativi neoliberisti che ne minacciano la sopravvivenza e ne tagliano le risorse (Knutas, 2017; Corbett, 2016; Kalaoja, Pietarinen, 2009). L'attenzione è altresì posta sull'atteggiamento, che possiamo chiamare scettico, con il quale si guarda a queste realtà scolastiche, considerate spesso scuole di serie B quando poste a confronto con le scuole di città. I termini di paragone sono i risultati dell'apprendimento costruiti su base metro-centrica (White, Corbett, a cura di, 2014; Beach et al., 2019) e standardizzata (ne sono esempio i test INVALSI, PISA, etc.). Questi termini di paragone non tengono conto delle caratteristiche proprie delle piccole scuole, come ad esempio la realtà rurale all'interno della quale sono inserite o l'organizzazione in pluriclasse per necessità. 
Molte delle scuole da noi visitate si trovano ogni anno in situazione precaria e spesso bambini, genitori ed insegnanti non sanno di anno in anno se la scuola rimarrà aperta. In una delle valli del Trentino, la Val di Fassa, I'ultima piccola scuola pluriclasse è stata chiusa nel 2016, I'anno prima dell'inizio della nostra ricerca. Una cosa è certa: nessuna di esse, nel momento in cui viene chiusa, riapre le porte.

Date queste premesse, lo scopo di questo lavoro di ricerca è quello di astrarre le proprietà essenziali (Charmaz, 2009) del rapporto tra le piccole scuole rurali e le comunità nelle quali esse sono inserite, per capire quali siano gli elementi che più di altri fanno di queste scuole esempi da portare al sistema educativo più ampio. Con i bambini al centro dell'attenzione, questo lavoro analizza l'interazione tra bambini, insegnanti, genitori, dirigenti, politici locali, residenti e famiglie straniere, così come la loro relazione con l'ambiente naturale. Seguendo i fondamenti della metodologia Grounded Theory (da qui in poi GT nel testo) sono state visitate 14 scuole primarie rurali italiane con almeno una pluriclasse, dal Trentino alla Sicilia passando per le Marche. Seguendo gli approcci della GT costruttivista e Informed (Charmaz, 2014; Thornberg, 2012) si sono costruiti alcuni concetti sensibilizzanti (Blumer, 1954) i quali hanno guidato la ricerca sul campo: il concetto di comunità rurale; il ruolo dell'insegnante nei contesti rurali; la pluriclasse per necessità; le politiche educative sulle piccole scuole. Attraverso la lente della pedagogia critica, una particolare attenzione è stata posta sul ruolo dell'insegnante nei contesti rurali (Corbett, 2016), visto come figura ponte tra cultura e comunità. L'attento sguardo a queste piccole realtà scolastiche offre una serie di spunti teorici rilevanti per i responsabili politici e gli insegnanti che si trovano a prendere decisioni o a apportare il loro contributo educativo in queste realtà. I concetti costruiti sulla base dell'analisi dei dati raccolti all'interno delle scuole e comunità rurali entrano altresì in dialogo e possono aprire la ricerca ai contesti di comunità locale delle città, anch'esse costituite da periferie e da comunità all'interno di un contesto più ampio.

\title{
La chiusura di una piccola scuola di montagna
}

\author{
La scomparsa del curato di campagna, del medico di \\ campagna, del maestro di campagna è il motivo sca- \\ tenante della dissoluzione delle comunità rurali. \\ Aldo Gorfer, giornalista e scrittore trentino
}

L'interesse di ricerca che guida questo lavoro sorge dalla chiusura della piccola scuola primaria pluriclasse di Pejo Paese, in Trentino sulle Alpi del nord Italia, nel 2011. Pejo Paese è un paesino di circa 400 abitanti, a 1585 metri s.l.m. ed è il più al to tra i centri abitati della regione. L'economia di questi luoghi si basa principalmente sul turismo estivo ed invernale e su piccole aziende agricolo-zootecniche. La valle di Pejo è famosa sin dal XVII secolo per le sue acque ferruginose, infatti poco più a valle esiste ancora il complesso termale, ristrutturato e rimesso in funzione sugli standard turistici dei nostri tempi, nonché lo stabilimento di imbottigliamento dell'acqua Pejo. Pejo Paese dista $10 \mathrm{~km}$ circa di strada a tornanti dal fondovalle, dove nel 2011 è sorto il nuovo complesso scolastico che riunisce i bambini e le bambine di tutti i centri abitati della valle. Ora i bambini e le bambine di Pejo Paese scendono ogni mattina a scuola con il bus.

La decisione riguardo alla chiusura della scuola è stata presa dalla Provincia Autonoma di Trento, d'intesa con il Dipartimento della Conoscenza della Provincia, organo territoriale amministrativo e di ricerca in materia di istruzione. È bene qui sottolineare 
come in Trentino, Provincia Autonoma, la scuola sia di tipo provinciale a carattere statale, il che significa che vi è un adeguamento a livello territoriale delle leggi nazionali. La scuola primaria di Pejo Paese era organizzata in un'unica pluriclasse, dalla prima alla quinta classe, e i bambini e le bambine frequentanti erano 15. Questo numero non corrispondeva al numero degli alunni in età scolare primaria residenti nel paese. Alcune famiglie preferivano già portare i propri bambini a fondovalle, chi per scetticismo nei confronti della pluriclasse, chi per l'offerta formativa più ampia (attività pomeridiane, mensa, etc.) che la piccola scuola di Pejo Paese non poteva garantire, chi ancora per la maggior vicinanza al luogo di lavoro o alla casa dei nonni. Un gruppo di genitori si oppose però più volte alla chiusura della scuola, cercando di convincere la Provincia a ritrattare la decisione. Dopo svariati rifiuti da parte del Dipartimento dell'Istruzione e degli assessori provinciali, questi stessi genitori decisero di intraprendere l'avventura di Scuola Pejo Viva, una scuola autogestita organizzata per motivi burocratici sull'istruzione parentale, prevista dalla Costituzione italiana, ma che ha poi visto insieme ai genitori la presenza di molti insegnanti e educatori a titolo gratuito (tra i quali il maestro che lì insegnava da nove anni). Il progetto vide come protagonisti nove bambini e bambine, al piano terra di una casa privata, senza Provincia Autonoma, Ministero della Pubblica Istruzione, Comune, dirigenti scolastici, burocrazia, scuolabus, voti, psicologi, orari di 30 ore settimanali, fotocopiatrice, etc. Questo per due anni scolastici, il tempo di portare i bambini di quarta e quinta all'esame di ammissione alla scuola secondaria di primo grado. L'esame fu egregiamente superato da tutti. Il progetto si è fermato qui. La storia di questo gruppo di genitori, maestri e professori che vedono la piccola scuola come elemento fondamentale per la comunità è raccontata nel documentario di Michele Trentini e curato da Giulia Mirandola, intitolato Alta scuola (Trentini, Mirandola, 2016; trailer disponibile alla pagina https://youtu.be/WooUcz9xctk). In Trentino Scuola Pejo Viva è stata il primo esperimento di scuola primaria autogestita nata dalla chiusura della scuola pubblica.

Come possiamo vedere nella tabella qui sotto, il Trentino, con il 2,9\% di pluriclassi supera leggermente la percentuale nazionale.

Tabella 1. Percetuale delle pluriclassi nella scuola primaria in Italia e in Trentino

\begin{tabular}{ccccc}
$\begin{array}{c}\text { Paesel } \\
\text { Provincia }\end{array}$ & $\begin{array}{c}\text { Numerototale di } \\
\text { classi }\end{array}$ & $\begin{array}{c}\text { Numerototale di } \\
\text { pluriclassi }\end{array}$ & \% dipluriclassi & Anno \\
\hline Italia & 133.304 & $1.400 \sim$ & $1 \sim$ & $2017 / 18$ \\
Trentino & 1.447 & 42 & 2,9 & $2017 / 18$ \\
\hline
\end{tabular}

Fonte: ISTAT, INDIRE e Dip. della Conoscenza (TN)

Tabella 2. Percentuale delle pluriclassi nella scuola primaria in Svizzera, Austria, Italia, Alto Adige e Trentino

\begin{tabular}{ccccc}
$\begin{array}{c}\text { Paesel } \\
\text { Provincia }\end{array}$ & $\begin{array}{c}\text { Numero totale di } \\
\text { classi }\end{array}$ & $\begin{array}{c}\text { Numero totale di } \\
\text { pluriclassi }\end{array}$ & \%dipluriclassi & Anno \\
\hline Svizzera & 22.361 & 5.371 & 24,0 & $2009 / 10$ \\
Austria & 17.899 & 2.735 & 15,3 & $2012 / 13$ \\
Italia & 133.304 & 1.400 & $1 \sim$ & $2017 / 18$ \\
Alto Adige & $266^{*}$ & $109^{* *}$ & 40,9 & $2014 / 15$ \\
Trentino & 1.447 & 42 & 2,9 & $2017 / 18$ \\
\hline
\end{tabular}

* Scuole, non classi ** Scuole con meno di 40 alunni 
Come possiamo osservare ancora nella tabella successiva, in alcuni Paesi al confine con I'Italia la percentuale delle pluriclassi nella scuola primaria è notevole: in Svizzera e Austria le pluriclassi sono rispettivamente il $24 \%$ e il $15,3 \%$ del totale delle classi (Smit, HyryBeihammer, Raggl, 2015). L'Italia non presenta una percentuale così significativa, contando I' $1 \%$ di pluriclassi. Un'interessante eccezione è rappresentata dalla Provincia di Bolzano, dove più del $40 \%$ delle scuole primarie presenti sul territorio contano meno di 40 alunni, da cui si può dedurre siano pluriclassi. Questa particolarità si può spiegare con il fatto che il Sudtirolo è trilingue: si parlano tedesco, italiano e ladino. Qui sono presenti tre diverse intendenze scolastiche, separate in base alla lingua, le quali intendono preservare le caratteristiche di ciascuna entità linguistica. A differenza della Provincia di Bolzano, il Trentino ha un unico sistema educativo, quello italiano.

Le piccole scuole esistono in tutto il mondo; si stima che le scuole organizzate in pluriclasse corrispondano al 30\% circa del totale delle scuole nel mondo (Boix Tomás, Domingo Peñafiel, 2018, p. 15). In Italia le piccole scuole sono dislocate in aree quali i piccoli villaggi di montagna, le piccole isole o le campagne dell'entroterra. In queste scuole il numero degli scolari può essere molto piccolo. Il Decreto del Presidente della Repubblica italiana approvato nel 2009 e centrato sulla riorganizzazione della rete scolastica, stabilisce il numero minimo di alunni in una scuola a otto (DPR, 20 marzo 2009 n. 81). Ciò nonostante, esistono piccole scuole primarie con meno di otto bambini, come sull'isola di Alicudi, nelle Eolie, dove durante l'anno scolastico 2017/18 si contavano soltanto tre alunni tra scuola primaria e secondaria di primo grado, organizzati in una pluriclasse (Cimatti, 2017). Oppure il caso della scuola primaria di Casatta-Valfloriana, in Trentino, da noi visitata nel 2018, dove sei tra bambini e bambine frequentano una pluriclasse dalla prima alla quinta.

\section{Metodologia: la Grounded Theory costruttivista e Informed}

Lo scopo di questo lavoro di ricerca è quello di astrarre le proprietà essenziali (Charmaz, 2009) del rapporto tra le piccole scuole rurali e le comunità nelle quali esse sono inserite. Sin dal primo approccio sul campo è emersa una caratteristica che ha dettato la scelta metodologica della ricerca: I'eterogeneità di queste realtà. Rispetto alla storia della scuola di Pejo Paese, sono apparse altre realtà, differenti per vari aspetti da questa. Diverse realtà economiche e sociali, ambientali, di infrastrutture e diversi approcci educativi e didattici, fanno delle piccole scuole un gruppo variegato. Non solo all'interno del Paese, dalle Alpi al Lilibeo, ma anche all'interno della stessa provincia o regione. Inoltre, il non voler isolare la realtà scolastica da quella della comunità nella quale è inserita, ha reso il fenomeno da studiare ancor più complesso. Da qui la scelta di adottare una metodologia che permettesse «il confronto fra elementi differenti e lontani» (Tarozzi, 2008, p. 15), caratteristica della GT.

Perciò quello che qui si propone è uno studio di tipo qualitativo che segue i fondamenti della GT (Glaser, Strauss, 1967) attraverso l'approccio costruttivista e etnografico promosso dalla Charmaz (Charmaz, 2014; Charmaz, Mitchell, 2014) e la versione Informed suggerita da Thornberg (2012). Questi due approcci hanno permesso di conoscere il significato che i partecipanti attribuiscono alla relazione tra piccola scuola e comunità, assicurando che sia il processo che il prodotto della ricerca rimangano «fondate nei dati attraverso i metodi della Grounded Theory, pur attingendo informazioni dalla letteratura di ricerca e dai quadri teorici esistenti» (Thornberg, 2012, p. 249). 
La natura della GT è quella di generare teoria: «La grounded theory si basa sul generare sistematicamente teoria dai dati, i quali sono essi stessi ottenuti attraverso la ricerca sociale» (Glaser, 1978, p. 2). Questo lavoro di ricerca non ha l'ambizione di produrre una teoria sociale. Si è cercato più modestamente di costruire e raggruppare temi e categorie, codificati attraverso l'analisi dei dati raccolti sul campo, sviluppandone i significati e descrivendone le relazioni. Lo scopo è quello di cercare le ricorrenze rintracciate all'interno dei dati raccolti e, sulla base di queste, costruire le categorie concettuali che consentano un'interpretazione della relazione che intercorre tra piccola scuola e comunità. Queste categorie verranno definite e ne verranno definite le relazioni. Verranno effettuate comparazioni tra le definizioni costruite sulla base di quanto emerso sistematicamente dal campo e analizzato attraverso i metodi della GT e altre definizioni dello stesso concetto presenti in letteratura.

II principale strumento per la raccolta dei dati usato in questo studio è l'intervista intensiva (Charmaz, 2014) con mestre/i delle pluriclassi, genitori, dirigenti scolastici e sindaci dei paesini a cui la scuola fa capo. Essi sono stati intervistati nelle Marche, in Trentino, e sulle due piccole isole di Stromboli e Marettimo in Sicilia. A questo strumento di raccolta dati si è affiancata l'osservazione etnografica (Charmaz, Mitchell, 2014; Tarozzi, 2008). Ci è parso fondamentale, sin dall'inizio della ricerca, poter osservare direttamente il lavoro in classe degli stessi insegnanti intervistati per avere uno strumento in più da mettere in relazione ai dialoghi emersi durante le interviste. Sono stati inoltre raccolti e analizzati alcuni documenti preesistenti (extant) (Charmaz, 2009) come fonte di dati complementare alle interviste intensive e alle osservazioni etnografiche. Oltre all'analisi delle leggi nazionali, regionali e comunali inerenti alle realtà delle piccole scuole in zone rurali, sono stati raccolti e analizzati progetti scolastici, attività, portfolios, articoli o riflessioni scritti da insegnanti o altre figure, regolamenti e statuti che regolano la vita della piccola scuola, nonché le proposte di legge.

Le interviste intensive con gli insegnanti hanno avuto luogo a scuola durante le ore libere dalle lezioni, prima o dopo l'osservazione in classe con gli stessi insegnanti. Sono stati intervistati 37 insegnanti, 9 genitori, 4 dirigenti scolastici e 5 sindaci. Le scuole in cui sono state effettuate le interviste sono 14 in totale: nove in Trentino, tre nelle Marche e due in Sicilia. La durata media delle interviste è stata di 60 minuti. La maggior parte delle interviste si sono svolte durante l'anno scolastico 2017/18. Nelle scuole di Rumo e di Casatta-Valfloriana siamo ritornati anche l'anno scolastico successivo 2018/19 per verificare le categorie e i concetti costruiti sulla base dei dati. In queste occasioni abbiamo intervistato anche nuovi soggetti (genitori, sindaci, dirigenti, etc.), abbiamo parlato di nuovo con gli insegnanti e osservato nuovamente le pluriclassi. Riguardo alla nostra presenza come ricercatori all'interno delle scuole italiane, si è dovuta richiederne l'autorizzazione ai dirigenti scolastici, figure responsabili della sicurezza e dell'organizzazione dei vari plessi scolastici. Il primo contatto di riferimento è stato l'indirizzo e-mail dei dirigenti dei vari istituti scolastici in cui si sapeva ricadesse la piccola scuola d'interesse. Nel caso in cui i dirigenti accogliessero la richiesta, l'incarico di mettere in relazione gli insegnanti con la ricercatrice veniva affidato al/alla coordinatore/trice di plesso o al/alla vicario/a. Da qui si passava al fissare un incontro con gli insegnanti delle singole scuole, di solito all'interno di un momento di programmazione. In questo spazio, la ricercatrice ha esposto i temi e lo scopo della ricerca e ci si è accordati con gli insegnanti sui giorni e gli orari delle osservazioni in classe e delle interviste. 
La scelta delle scuole da visitare si è basata su uno dei fondamenti della GT, il campionamento teorico. Esso è iniziato con le piccole scuole dell'Istituto comprensivo di Caldarola, nelle Marche e si è costruito a mano a mano che la ricerca procedeva, attraverso la ricerca delle scuole con pluriclassi presso le istituzioni scolastiche nazionali (MIUR) e provinciali (Dipartimento della Conoscenza di Trento), grazie a contatti personali, o ancora con il metodo a palla di neve (snowballing) (Biernacki, Waldorf, 1981). Con le parole di Glaser e Strauss

Il campionamento teorico è un processo di raccolta di dati in cui il ricercatore contemporaneamente raccoglie codifica e analizza i propri dati e, in base a ciò, decide quali ulteriori dati raccogliere e dove trovarli, al fine di sviluppare la teoria nel momento in cui emerge. (Glaser, Strauss, 2009, p. 75)

Già dai primi codici e codici in-vivo, assegnati alle interviste e ai documenti raccolti durante le visite alle scuole nelle Marche, sono emerse interessanti categorie, le quali hanno guidato la scelta delle successive scuole da visitare.

Tabella 3. Campionamento teorico

\begin{tabular}{cc} 
Regione & Scuole \\
\hline Marche & Cessapalombo, Camporotondo, Serrapetrona \\
\hline Trentino & $\begin{array}{r}\text { Rumo, Lases, Sover, Casatta-valfloriana, Masi di cavalese, } \\
\text { Capriana, Ospedaletto, Samone, Nomi }\end{array}$ \\
\hline Sicilia & Stromboli, Marettimo \\
\hline
\end{tabular}

Nel caso del Trentino, regione di residenza della ricercatrice, si è proceduto richiedendo al Dipartimento della Conoscenza un elenco delle scuole all'interno delle quali fosse presente almeno una pluriclasse e da qui si è passati a contattare con una e-mail i dirigenti scolastici delle scuole aventi caratteristiche interessanti per la nostra ricerca. Abbiamo contattato in Trentino tutti i 16 istituti comprensivi della lista, ricevendo risposta affermativa alla nostra presenza nelle scuole da cinque di essi. In questo modo abbiamo visitato 9 delle 27 scuole con pluriclassi della Provincia.

Tabella 4. Scuole visitate in Trentino, organizzazione delle pluriclassi e numero di insegnanti intervistati

\begin{tabular}{|c|c|c|c|}
\hline Scuole & $\begin{array}{c}\text { Totale dei } \\
\text { bambini }\end{array}$ & $\begin{array}{l}\text { Numero di bambini per classe e } \\
\text { organizzazione delle pluriclassi }\end{array}$ & $\begin{array}{l}\text { Insegnanti } \\
\text { intervistati } \\
\end{array}$ \\
\hline Nomi & 47 & $1^{\mathrm{a}}+2^{\mathrm{a}}(3+9) ; 3^{\mathrm{a}}(5) ; 4^{\mathrm{a}}(20) ; 5^{\mathrm{a}}(10)$ & 2 \\
\hline Rumo & 44 & $1^{\mathrm{a}}(9) ; 2^{\mathrm{a}}+3^{\mathrm{a}}(7+6) ; 4^{\mathrm{a}}(13) ; 5^{\mathrm{a}}(9)$ & 2 \\
\hline Masi di Cavalese & 39 & $1^{\mathrm{a}}+2^{\mathrm{a}}(8+7) ; 3^{\mathrm{a}}(11) ; 4^{\mathrm{a}}+5^{\mathrm{a}}(6+7)$ & 4 \\
\hline Ospedaletto & 35 & $1^{a}(10) ; 2^{a}+3^{a}(4+5) ; 4^{a}+5^{a}(6+10)$ & 3 \\
\hline Samone & 34 & $1^{\mathrm{a}}+2^{\mathrm{a}}(9+9) ; 3^{\mathrm{a}}+4^{\mathrm{a}}(10+6) ; 5^{\mathrm{a}}($ zero) & 2 \\
\hline Lases & 29 & $1^{\mathrm{a}}+2^{\mathrm{a}}+3^{\mathrm{a}}(2+1+10) ; 4^{\mathrm{a}}+5^{\mathrm{a}}(12+4)$ & 4 \\
\hline Capriana & 28 & $1^{a}+2^{a}+3^{a}(6+6+5) ; 4^{a}+5^{a}(3+8)$ & 3 \\
\hline Sover & 25 & $1^{a}+2^{a}+3^{a}(3+3+5) ; 4^{a}+5^{a}(8+8)$ & 4 \\
\hline Casatta-Valfloriana & 6 & $1^{\mathrm{a}}\left(\right.$ zero); $2^{\mathrm{a}}$ (zero); $3^{\mathrm{a}}+4^{\mathrm{a}}+5^{\mathrm{a}}(1+3+2)$ & 3 \\
\hline
\end{tabular}


Gli elementi che hanno guidato il campionamento teorico durante l'arco della ricerca sono stati:

1. La presenza di organizzazioni a livello locale, attive sul territorio a vari livelli, dalle quali si potesse desumere l'esistenza di un senso di comunità all'interno del villaggio in cui la scuola è inserita.

2. La presenza e l'intervento di figure motivate a far sì che la scuola diventi il centro della comunità, ad usare gli spazi della scuola come spazi di incontro per la comunità tutta.

3. La presenza di insegnanti all'attivo da diversi anni nella stessa piccola scuola, i quali avessero una conoscenza approfondita del luogo, dei suoi abitanti, della loro storia e dell'ambiente circostante.

4. La presenza di condizioni geografiche o climatiche estreme che mettono il villaggio in cui la scuola è inserita in uno stato di isolamento a causa della difficoltà nel raggiungere questi luoghi.

5. La presenza di famiglie con background migratorio.

6. La presenza di un'unica pluriclasse dalla classe prima alla quinta e, conseguentemente, un numero veramente esiguo di bambini e bambine frequentanti la piccola scuola.

7. La presenza di attività che mettono in relazione la scuola con la comunità progettate dagli insegnanti e/o da altre figure o enti locali.

8. La presenza di una volontà politica volta alla sopravvivenza della piccola scuola del villaggio.

Oltre a guidare il campionamento teorico, questi stessi elementi hanno guidato la comparazione tra le diverse scuole e villaggi. Le interviste sono state registrate e le loro trascrizioni sono state poi codificate usando il software MAXQDA2018. Si è quindi proceduto attraverso la codifica aperta (open coding), la quale avviene attraverso la destrutturazione del testo in frammenti di dati (fragments of data) - parole, linee, segmenti e incidenze - (Charmaz, 2014), facendo risaltare anche i piccoli dettagli, le sfumature delle singole parole usate, le quali anch'esse possono generare codici fondamentali. La codifica accadimento (incident) per accadimento (Glaser, 1978) è stata utilizzata per aprire i dati ed esplorare frammenti di testo per rivelare tutti i possibili significati che il testo poteva generare (Tarozzi, 2008). Si è cercato in questa prima fase di analisi di mantenere la nominazione dei codici molto vicina alle stesse parole usate dai partecipanti (in-vivo codes). La codifica aperta è quindi rimasta strettamente legata ai dati e non ha applicato categorie preesistenti. Alla fine della codifica aperta avevamo 142 codici assegnati.

La fase di codifica aperta si è conclusa nel momento in cui tutti i dati hanno trovato posto all'interno dei codici e i codici stessi si sono focalizzati attorno a particolari problemi, «riempiendoli di tutti i significati possibili che il ricercatore è riuscito ad assegnare loro» (Glaser, 1978, p. 56). II livello successivo è stato quello della codifica focalizzata (focused coding), la quale segna il momento del passaggio al livello dell'astrazione, «il momento dell'abbandono del piano descrittivo per quello concettuale. [...] Lo scopo della codifica focalizzata è quello di raccogliere i concetti in categorie e individuare concetti a un più elevato livello di astrazione, ma è anche il livello in cui si collegano fra loro le cate- 
gorie e queste con le loro proprietà» (Tarozzi, 2008, p. 93). Abbiamo costruito le macrocategorie e le loro sottocategorie sulla base dei 142 codici della codifica aperta attraverso un'operazione di clustering (Charmaz, 2014, pp. 184 e seg.), cioè accorpando i codici in categorie più ampie che li contengano. Come riassunto nella tabella qui sotto, in questa fase dell'analisi si è passati dai 142 codici iniziali alla creazione di 6 macrocategorie con le relative sottocategorie.

Tabella 5. Macrocategorie, sottocategorie e proprietà costruire durante la fase di codifica

Macrocategorie

\begin{tabular}{cc}
$\begin{array}{c}\text { Piccola scuola come motore per lo svi- } \\
\text { luppo della comunità }\end{array}$ & $\begin{array}{c}\text { Cooperazione } \\
\text { Responsabilità } \\
\text { Autonomia }\end{array}$ \\
\hline $\begin{array}{c}\text { Ruolo dell'insegnante come intellettuale } \\
\text { organico }\end{array}$ & $\begin{array}{c}\text { Autonomia del lavoro dell'insegnante } \\
\text { Didattica dell'esperienza }\end{array}$ \\
\hline Piccola scuola come famiglia & $\begin{array}{c}\text { Creazione di un percorso educativo circolare } \\
\text { Partecipazione dei genitori alla vita scolastica } \\
\text { Sinergia }\end{array}$ \\
\hline Formalismo del sistema scolastico & $\begin{array}{c}\text { Omogeneizzazione dell'insegnamento } \\
\text { Burocratizzazione del sistema scolastico } \\
\text { Tecnicismo del ruolo dell'insegnante } \\
\text { Valutazione su scala metro-centrica } \\
\text { Scetticismo sulla piccola scuola }\end{array}$ \\
\hline Comunità reale & Integrazione nelle comunità locali \\
Convivialità
\end{tabular}

Oltre allo strumento visuale del clustering, questa operazione di collegamento e individuazione delle proprietà essenziali delle categorie è stata possibile grazie alla scrittura dei memos, note che il ricercatore scrive e attraverso le quali «fissare i ragionamenti, catturare le comparazioni e connessioni che si fanno e cristallizzare domande e indicazioni future da seguire» (Charmaz, 2014, p. 162). Essi hanno permesso di definire le connessioni tra le macrocategorie, collegandole tra loro e con le sottocategorie.

Si è passati poi alla codifica teorica (theoretical coding), attraverso la quale «si precisano e si qualificano le relazioni che sussistono fra le categorie che sono emerse dalla codifica focalizzata» (Tarozzi, 2008, p. 52). Questo è anche il passaggio che definisce la categoria centrale (core-category), cioè «il concetto organizzatore principale di un'area di ricerca» (Tarozzi, 2008, p. 100).

Il diagramma che segue illustra la definizione della categoria centrale e la relazione tra le varie categorie. Abbiamo identificato il concetto di community education come categoria centrale. Questo concetto è collegato direttamente al codice in-vivo «piccola scuola come motore per lo sviluppo della comunità», ma ne amplia i confini, dato che vede la relazione tra la scuola e la comunità bidirezionale. Se la scuola può essere motore per lo sviluppo della comunità, la comunità può essere altresì motore per lo sviluppo dell'educazione, come è emerso dall'analisi dei dati raccolti. Questo concetto mantiene quindi il ruolo centrale della scuola vista come motore per lo sviluppo della comunità, ma 
apre l'interpretazione del fenomeno studiato anche nell'altra direzione. Da qui la sua forza esplicativa.

Figura 1. La categoria centrale community education e le macrocategorie che formano le caratteristiche essenziali della relazione tra la piccola scuola e la comunità

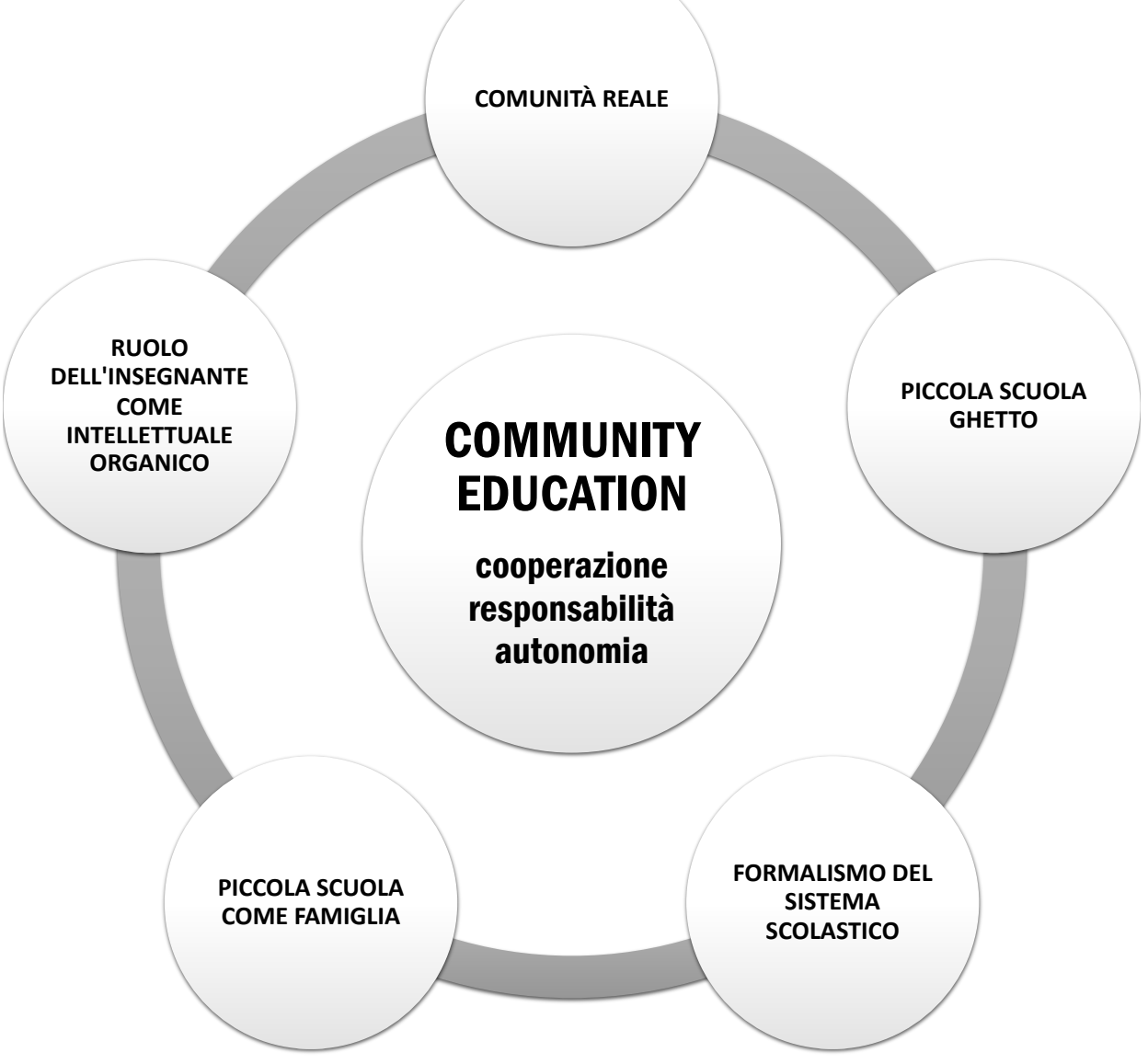

Vediamo come questo modello preveda la categoria centrale come nucleo delle sottocategorie che le ruotano intorno e come esse siano a loro volta interconnesse tra loro.

\section{Community education: la piccola scuola motore per lo sviluppo della comunità}

Un primo dato generale emerso dalla ricerca è l'eterogeneità delle realtà visitate. Le piccole scuole si sono presentate agli occhi del ricercatore molto diverse l'una dall'altra sotto vari aspetti: socio-economici, ambientali, di infrastrutture e, all'interno di questi, abbiamo trovato diversi approcci educativi e didattici. Con l'andare avanti della ricerca e grazie all'analisi dei dati raccolti sul campo, è emerso però in maniera sempre più esplicita un elemento che trascende questa eterogeneità e cioè la presenza di figure fortemente motivate (insegnanti, genitori, dirigenti scolastici o sindaci) che vedono la scuola come motore per lo sviluppo della comunità e che agiscono in questo senso. Attorno alle azioni di queste figure all'interno della vita scolastica delle realtà visitate, si è costruito il concetto chiave di community education.

II significato e l'uso di questo termine abbraccia non solo gli ambienti scolastici, ma apre il discorso sull'educazione a tutta la comunità che in un determinato luogo vive o di 
quel luogo fa in qualche modo esperienza. In altre parole, questo processo educativo comunitario non arricchisce solo i bambini e le bambine della scuola, ma va oltre le mura della classe e investe tutta la comunità, facendo partecipare anch'essa al processo educativo in un'ottica di Lifelong Learning. Il processo va quindi visto in entrambe le direzioni.

Figura 2. Il sistema bidirezionale tra scuola e comunità

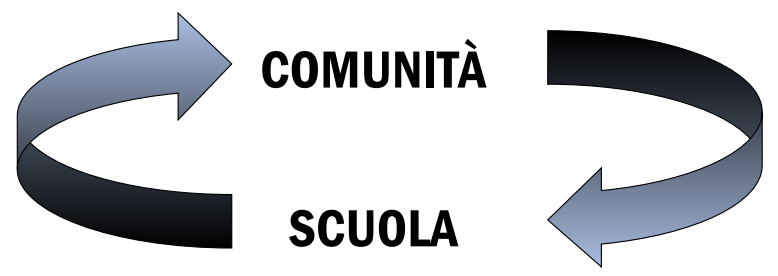

Dalle definizioni che del concetto di community education sono state date (Beneke, 2016; Hüther, 2013; Westoby, Shevellar, 2012; Buhren, 1997) si possono estrapolare alcuni elementi comuni: la presenza di uno spazio comune, la soddisfazione del bisogno di interazione sociale e il miglioramento della vita comunitaria e sociale. Oggi il concetto di community education si muove su due binari, teorico e pratico (Westoby, Shevellar, 2012, p. 4). Dal primo si traggono consapevolezza e conoscenze, dal secondo l'azione che si basa sempre e comunque su concetti teorici, come quello di democrazia. Così, attraverso il concetto di democrazia e lo sforzo pragmatico nel mettere in atto i suoi principi, veniamo a parlare dell'autore e pensatore al quale più di tutti la community education fa riferimento: John Dewey. Il concetto di community education nasce dal solco del pensiero di Dewey, secondo il quale la scuola deve essere innanzitutto un centro per lo sviluppo sociale e democratico (Buhren, 1997, p. 18 e seg.). Nonostante il pensiero di Dewey comprenda la società nel senso più ampio e sia stato scritto all'inizio del ' 900 , esso è valido anche per le più piccole comunità e è sempre attuale, come dimostra l'interesse in ambito educativo mai tramontato per il pensiero di questo grande filosofo. In Democrazia e educazione, Dewey scriveva:

\footnotetext{
Una società indesiderabile [...] è una società che, internamente ed esternamente, crea barriere al libero scambio e alla comunicazione dell'esperienza. Democratica è invece una società che preveda la partecipazione al bene di tutti i suoi membri in condizioni di parità e che assicuri un riadattamento flessibile delle sue istituzioni attraverso l'interazione delle diverse forme di vita associata. Una società di questo tipo deve avere un tipo di educazione che dia agli individui un interesse personale nelle relazioni sociali e nel loro controllo, nelle abitudini mentali che assicurano cambiamenti sociali senza introdurre disordine. (Dewey, 1997, p. 99)
}

II concetto di community education non può essere oggi spiegato se non mettendolo in relazione alle politiche economiche su scala mondiale ed è strettamente legato a quello di trasformazione a livello economico-sociale, usato qui nel suo significato radicale proposto dalla Elsen:

\footnotetext{
La terza 'Grande trasformazione', quella eco-sociale, in contrasto con la prima 'Grande trasformazione' (la trasformazione neolitica) e la seconda (la Rivoluzione Industriale), non sarà il risultato di un processo sociale evolutivo. Deve essere un cambio riflessivo e pianificato di quasi tutti i pilastri delle società industriali moderne: della produzione, del consumo, degli stili di vita socioculturali e individuali. (Elsen, 2019, p. 5)
}

Il concetto di community education, come quello di community economy, porta quindi con sé l'idea di un cambio, il cui scopo è quello di inserire nuovamente l'educazione dentro i suoi contesti socioculturali e naturali (ivi, p. 26), facendo in modo che questi diventino il teatro delle esperienze sulle quali costruire la conoscenza. Dal punto di vista delle esperienze educative crediamo vi sia una tensione tra due visioni sul ruolo della 
scuola, le quali sono influenzate dai processi globali che regolano le nostre vite economiche e sociali.

Diversi luoghi offrono possibilità e contesti diversi di indirizzo della comunità locale a partire dall'educazione, non per ultimo a causa delle condizioni economiche e delle relazioni produttive predominanti e della crescente globalizzazione basata su una specifica forma di capitalismo e di economia. (Beach et al., 2019, p. 2)

Così, da un lato abbiamo una visione di educazione che ha come fine quello trasformativo/emancipativo e, dall'altro, un'idea di educazione che ha come obiettivo quello di realizzare il progetto neoliberista. Nel primo caso, la scuola tenderà ad essere un luogo in cui le persone si trattano come uguali, con dignità, per produrre una società che sostenga ogni individuo nel suo pieno sviluppo come essere umano. Essa porterà ad una società in cui ciascuno collabora per il reciproco vantaggio. Sarà quella società democratica di cui parlava Dewey. Nel secondo caso, la scuola tenderà a produrre una società organizzata per promuovere la competizione tra le persone, per separare le persone in leaders e masse, le quali devono essere controllate in maniera gerarchica e rese utili per lo sviluppo del progetto economico (Schostak, 2016). Questo tipo di scuola porterà ad una società dell'individualismo competitivo in cui solo pochi raccolgono i frutti della società nell'interesse delle tendenze economiche. II neoliberismo, come forza globale, ha favorito il flusso di ricchezza e potere nelle mani di pochi: secondo il rapporto del 2019 di Oxfam, dal titolo Public good or private wealth?, l'82\% dell'incremento di ricchezza globale va nelle mani dell' $1 \%$ della popolazione e, per rimanere in ambito educativo, 262 milioni di bambini e bambine non vanno a scuola (Oxfam, 2019). In più il modello neoliberista ha trasferito l'attività economica dalla piccola alla grande scala, così come ha soppiantato i prodotti artigianali e locali con quelli finanziari. Quali sono le implicazioni di questi meccanismi per la piccola scuola rurale? Essenzialmente il potere viene tolto dalle mani degli attori rurali. In opposizione a questo, un progetto basato sulla community education ha come obiettivo quello di reinserire l'educazione a vari livelli nelle mani della comunità locale. Questa «ridistribuzione è una delle più importanti strategie della trasformazione eco-sociale per la sostenibilità» (Elsen, 2019, p. 26).

Una scuola che promuove tra le sue attività la conoscenza del territorio e dei suoi abitanti, la storia del luogo e la sua economia, le possibilità che oggi questi luoghi offrono alla vita affettiva e lavorativa e che lo fa sviluppando la giustizia sociale attraverso l'educazione cooperativa, democratica e sostenibile, questa è una scuola che ha come fine lo sviluppo della comunità all'interno della quale è inserita.

In particolare, tre sono le proprietà che ruotano attorno alla categoria centrale di community education e che ne formano la struttura educativa: cooperazione, responsabilità e autonomia sono le tre proprietà.

Il concetto di community education è il nucleo del nostro modello esplicativo, attorno al quale ruotano altre categorie principali. Ci soffermeremo qui in particolare solo su quella di «ruolo dell'insegnante come intellettuale organico», perché essa si rivela essere la categoria di più interessante approfondimento.

\section{II ruolo dell'insegnante come intellettuale organico}

L'insegnante è intellettuale organico nel momento in cui favorisce la simbiosi tra la scuola e la comunità. Questa simbiosi è favorita da una serie di elementi che abbiamo costruito partendo sempre dai dati raccolti e che si possono concettualizzare nelle sotto-categorie: 
«autonomia del lavoro dell'insegnante» e «didattica dell'esperienza». Il concetto di «autonomia del lavoro dell'insegnante» è una sottocategoria di quello di "ruolo dell'insegnante come intellettuale organico» visto come possibilità di realizzazione dell'insegnante come professionista autonomo, in grado di appropriarsi del suo ruolo di intellettuale «attivamente coinvolto nella produzione di materiali didattici adeguati al contesto culturale e sociale in cui insegna» (Giroux, 1988, p. 125). Il riconoscimento fondamentale dell'autonomia del lavoro dell'insegnante è il primo elemento sul quale porre l'attenzione. Ci sembra interessante riportare qui il fatto che Vygotskij lavorò, nel periodo in cui Lenin fu al potere, all'interno di un sistema scolastico statale in cui non fu imposto un curriculum prestabilito, ma in cui veniva lasciata libertà agli insegnanti di costruire un percorso formativo che incontrasse le esigenze dei bambini a livello locale (Dixon-Krauss, a cura di, 2000, p. 21). La figura dell'insegnante diventa allora fondamentale per la creazione di una scuola che sa aprire le porte alla comunità e, allora come oggi, che fa fronte alla dispersione scolastica soprattutto nelle zone periferiche. Pensare di poter decontestualizzare l'insegnamento dall'ambiente in cui i bambini vivono la loro quotidianità significherebbe non tenere conto delle problematiche della comunità in cui la scuola è inserita, delle condizioni socio-economiche in cui le famiglie si trovano ad attuare. La figura dell'insegnante diventa allora fondamentale per imparare a guardare ai fenomeni sociali del mondo in cui viviamo in maniera critica come cittadini attivi. II ruolo dell'insegnante si eleva da quello di mero dispensatore di sapere, spesso stretto in un curriculum che non tiene conto della complessità dentro e fuori la classe.

Questa complessità è ancor più accentuata all'interno dell'organizzazione in pluriclassi tipica delle piccole scuole. Maria Montessori riteneva che la funzione principale dell'insegnamento fosse quella di valorizzare e rafforzare il potenziale individuale e che un'educazione uguale per tutti non fosse in grado di soddisfare questa richiesta (Montessori, 1947, 2014a). Sappiamo tutti che ogni persona è diversa. Ognuno di noi ha doni e talenti unici. Le nostre competenze e capacità possono essere molto diverse, da qui la necessità di un nuovo approccio educativo che sia in grado di sostenere contemporaneamente le diverse esigenze e attitudini dei bambini e che veda un valore nella diversità. Per il raggiungimento di questo obiettivo è necessario il riconoscimento dell'autonomia del lavoro dell'insegnante e che questo venga supportato dalle istituzioni educative (Tomazzolli, Consalvo, 2018).

Per tutti i motivi visti finora, il concetto di «autonomia del lavoro dell'insegnante» è strettamente legato a quello di «didattica dell'esperienza», nella misura in cui un tipo di approccio educativo basato sull'insegnamento e apprendimento attraverso progetti a livello comunitario ha bisogno di una certa libertà di azione. Progetti di questo tipo vanno costruiti con la comunità, con i genitori e altre figure di supporto come dirigenti, sindaci, organizzazioni. Essi sono autentici, non vanno bene in tutti i contesti allo stesso modo. Pensare di poter decontestualizzare l'insegnamento dall'ambiente in cui i bambini vivono la loro quotidianità significherebbe non tenere conto delle problematiche della comunità in cui la scuola è inserita, delle condizioni socio-economiche in cui le famiglie si trovano ad attuare. La figura dell'insegnante diventa allora fondamentale per imparare a guardare ai fenomeni sociali del mondo in cui viviamo in maniera critica come cittadini attivi. 
La «didattica dell'esperienza» ha alla sua base un progetto educativo ispirato ad una filosofia che ha radici nell'esperienza della vita (Dewey, 2014) e che perciò non eleva barriere tra la vita scolastica e la vita quotidiana del bambino. II motto di questo tipo di didattica è «partire dal mondo dei bambini», cioè costruire un'educazione che veda elevate ad esperienze quotidiane le attività che legano la scuola e la vita quotidiana perché esse possano convertirsi in pedagogia. Il lavoro dell'insegnante sarà quello di creare un senso logico tra ciò che accade nel mondo e il qui e ora, tra cosmo e focolare (Tuan, 2003), in un'ottica ecologica e complessa. Da qui la necessità di un approccio didattico interdisciplinare, in modo da approfondire gli interessi dei bambini passando, di volta in volta, attraverso la storia, la geografia, la matematica, le scienze, l'arte, il racconto, la lettura, il movimento e la grammatica. L'idea è quella di rielaborare le conoscenze acquisite partendo dalla realtà per poi consegnarle di nuovo al mondo. Tutte quelle attività che abbiamo visto attuate nelle piccole scuole visitate (giornalini, prodotti agricoli, lavori al traforo, cucito o preparazione di cibi), le quali vedono l'uso della mano e della mente insieme, portano alla creazione di qualcosa che viene rimesso in circolo. L'insegnante che si fa intellettuale organico inserisce queste attività in percorsi più complessi e interdisciplinari, in modo che esse non rimangano disgiunte e frazionate in sporadiche iniziative da attuarsi durante le ore facoltative. Egli cerca con i bambini il filo logico che tiene insieme la complessità, cosicché «lo sviluppo dell'attitudine a contestualizzare e globalizzare i saperi diviene un imperativo dell'educazione» (Morin, 2000, p. 19).

In quest'ottica educativa un ruolo importante riveste la natura, la relazione che instauriamo con essa. I geografi umani chiamano «approccio relazionale ibrido» quello che «combina le prospettive sociale e naturale con le quali le persone hanno familiarità [...] in una combinazione creativa» (Murdoch, in Woods, 2011, p. 41). Quindi, oltre all'importanza delle relazioni sociali che, come abbiamo visto, creano spazi e luoghi, l'enfasi è posta allo stesso modo sulla relazione con la natura, in un'ottica ecologica.

Mettere la vita all'interno dell'apprendimento didattico significa riconoscere che l'esperienza nel quotidiano costituisce un importante spazio per imparare. L'apprendimento avviene così nel contesto.

\section{Conclusioni}

Le relazioni non hanno luogo nello spazio, esse fanno lo spazio - esse compongono gli spazi relazionali e la geografia del mondo.

John Wylie, geografo umano

Il primo dato generale emerso dalla ricerca è l'eterogeneità delle scuole visitate e delle comunità nelle quali sono inserite. Esse si sono infatti presentate agli occhi del ricercatore molto diverse le une dalle altre sotto vari aspetti: socio-economici, ambientali, di infrastrutture e, all'interno di questi, abbiamo trovato nelle scuole diversi approcci educativi e didattici.

Con l'andare avanti della ricerca e grazie all'analisi dei dati raccolti sul campo, è emerso però in maniera sempre più esplicita un elemento che trascende questa eterogeneità e cioè la presenza di figure fortemente motivate (insegnanti, genitori, dirigenti scolastici, sindaci, associazioni e enti locali) che vedono la scuola come motore per lo sviluppo della comunità e che agiscono in questo senso. Particolare attenzione è stata posta 
nell'analisi sulla figura dell'insegnante. Da questa analisi si è costruito il concetto di «insegnante come intellettuale organico» per definire l'insegnante che vive lui stesso la comunità, ma con l'occhio della ricerca storica, letteraria, naturale, musicale e con tutto I'ambiente. Le esperienze raccolte durante il viaggio di ricerca traducono nella pratica concreta e quotidiana l'importanza del ruolo di ponte che l'insegnante viene ad assumere fra cultura e comunità.

La conclusione generale che possiamo trarre dalla ricerca qui presentata è che non sono principalmente i fattori strutturali dati dall'ambiente geografico o quelli demografici o ancora le risorse economiche a determinare la riuscita educativa nei contesti di piccola scuola rurale. In una prospettiva di community education, sono piuttosto le relazioni che intercorrono tra le famiglie, i bambini, gli insegnanti, i politici, le dirigenze scolastiche e gli enti locali a determinare la sopravvivenza e la bontà delle piccole scuole rurali. In questi casi la piccola scuola diventa uno spazio sociale dove i bambini sperimentano la partecipazione sociale e si formano ai valori democratici e cooperativi. Le politiche educative e i decisori politici potrebbero trarre beneficio dalla prospettiva che deriva da un attento sguardo a queste piccole forme di vita scolastica.

\section{Referimenti}

Aberg-Bengtsson, L. (2009) «The Smaller the Better? A Review of Research on Small Rural Schools in Sweden». International Journal of Educational Research, 48(2), pp. 100-108, https.//doi.org/10.1016/j.ijer.2009.02.007.

Beach, D.; Johansson, M.; Öhrn, E.; Rönnlund, M.; Per-Åke, R. (2019) «Rurality and Education Relations. Metro-Centricity and Local Values in Rural Communities and Rural Schools». European Educational Research Journal, 18(1), pp. 19-33. DOI. 1474904118780420.

Beneke, E. (2016) Community Education - How Communities Change Through Learning. Reading Online, ERASMUS+ Project «Community Education Facilitating». European Commission. Recuperat de: https://epale.ec.europa.eu/it/node/25806 [accés: 20/12/2019].

Biernacki, P.; Waldorf, D. (1981) «Snowball sampling. Problems and Techniques of Chain Referral Sampling». Sociological Methods \& Research, 10(2), pp.141-163.

Blumer, H. (1954) «What is Wrong with Social Theory? ». American Sociological Review, 18, pp. 3-10.

Boix Tomás, R.; Domingo Peñafiel, L. (2018) «La escuela rural, una mirada intercontinental». Aula de innovación educativa. Educación rural, una mirada intercontinental, 270, pp. 14-17.

Buhren, G.C. (1997) Community Education. Lernen für Europa. Münster-New York, Waxmann.

Charmaz, K. (2014) Constructing Grounded Theory. A Practical Guide Through Qualitative Analysis. London, SAGE.

Charmaz, K. (2009) «Shifting the Grounds. Constructivist Grounded Theory Methods», a Morse et al. (Eds.), Developing Grounded Theory, The Second Generation. Walnut Creek, CA., Left Coast Press.

Charmaz, K.; Mitchell, R.G. (2014) «Grounded Theory in Ethnography», a Atkinson, P.; Coffey, A.; Delamont, S.; Lofland, J. \& Lofland, L. (Eds.) Handbook of Ethnography. Los Angeles-London-New Delhi-Singapore-Washington DC., SAGE.

Cimatti, F. (2017, 21 novembre) La scuola più piccola d'Europa non trova maestri? Listening Online: Fahreneit, RaiRadio3 Podcast. Recuperat de: 
http.//rai.it/dl/portaleRadio/media/Contentltem-10a8bfaf-e0a9-4dbf-bc24c94de0b43d98.html [accés: 03/04/2018]

Corbett, M. (2016). «Reading Lefebvre from the Periphery. Thinking Globally About the Rural», a Schulte, A.K. \& Walker-Gibbs, B. (Eds.), Self-studies in Rural Teacher Education, Self-Study of Teaching and Teacher Education Practices, (14), Switzerland, Springer International Publishing, pp. 141-156.

Decreto del Presidente della Repubblica italiana 20 marzo 2009 n. 81. Norme per la riorganizzazione della rete scolastica e il razionale ed efficace utilizzo delle risorse umane della scuola, Gazzetta Ufficiale n. 151 (2-7-2009).

Dewey, J. (1915) Scuola e società. Firenze, La Nuova Italia (1987).

Dewey, J. (1916) Democracy and Education. New York, The Free Press (1997).

Dewey, J. (1938) Esperienza e educazione. Milano, Raffaello Cortina (2014).

Dipartimento della Conoscenza. Provincia Autonoma di Trento. Reading Online. Recuperat de: dip.conoscenza@provincia.tn.it [accés: 10/20/2017].

Dixon-Krauss, L. (Ed.) (2000) Vygotskij nella classe. Potenziale di sviluppo e mediazione didattica. Trento, Erickson.

Elsen, S. (2019) Eco-social Transformation and Community-Based Economy. New York, Routledge.

Giroux, H.A. (1988) Teachers as Intellectuals. Toward a Critical Pedagogy of Learning. Westport (CO), Bergin and Carvey, Greenwood Press.

Glaser, B.G. (1978) Theoretical Sensitivity. Advances in the Methodology of Grounded Theory. Mill Valley (CA), Sociology Press.

Glaser, B.G.; Strauss, A.L. (2009) La scoperta della Grounded Theory: strategie per la ricerca qualitativa. A. Strati (Ed.), Armando, Roma (ed. or. Glaser, B.G., \& Strauss, A.L. 1967. The Discovery of Grounded Theory. Strategies for Qualitative Research. New York, Hawthorne).

Gorfer, A. (1970) Solo il vento bussa alla porta. Trento, Arti Grafiche Saturnia,.

Hüther, G. (2013) Kommunale Intelligenz. Potenyialentfaltung in Städten und Gemeinden. Hamburg, Körber-Stiftung.

INDIRE. Manifesto delle piccole scuole: piccole scuole, trame sociali in rete. Reading Online. Recuperat de: http.//www.indire.it/progetto/piccole-scuole/ [accés: 25/09/2019].

ISTAT Italia. Reading Online. Recuperat de: http.//dati.istat.it/Index.aspx?Queryld=25455 [accès: 25/09/2018].

Kalaoja, E.; Pietarinen, J. (2009) «Small Rural Primary Schools in Finland. A Pedagogically Valuable Part of the School Network». International Journal of Educational Research, 48(2), pp. 109-116. DOI. 10.1016/j.ijer.2009.02.003.

Knutas, A. (2017) «Nordic Education Policy in Retreat Neoliberal Economic Rationalization and the Rural School». SAGE Policy Futures in Education, 0(0), pp.1-18. DOI. $10.1177 / 1478210317716307$.

Legge 13 luglio 2015 n. 107, Riforma del sistema nazionale di istruzione e formazione e delega per il riordino delle disposizioni legislative vigenti (La buona scuola), Gazzetta Ufficiale Serie Generale n.162 (15-07-2015).

Montessori, M. (1947) Come educare il potenziale umano. Milano, Garzanti (2014a).

Montessori, M. (1949) La mente del bambino. Mente assorbente. Milano, Garzanti (2014b).

Morin, E. (2000) La testa ben fatta. Riforma dell'insegnamento e riforma del pensiero. Milano, Raffaello Cortina.

Núñez-Muñoz, C.G.; Solís-Araya, C.; Soto-Lago, R. (2014) «¿Qué sucede en las comunidades cuando se cierra la escuela rural? Un análisis psicosocial de la política de cierre de las escuelas rurales en Chile». Universitas Psychologica, 13(2), pp. 615-625. DOI. 10.11144/Javeriana.UPSY13-2.qscc. 
Oxfam International Ong (2019) «Public Good or Private Wealth?». Reading Online: Oxfam International, UK. DOI. 10.21201/2019.3651. Recuperat de: https://indepth.oxfam.org.uk/public-good-private-wealth/ [accés: 03/01/2020].

Schostak, J. (2016) «Leaders, Leadership and Democracy - are they compatible?». Management in Education, 30(1), pp. 4-9. DOI. 10.1177/0892020615620750.

Smit, R.; Hyry-Beihammer, E. K.; Raggl, A. (2015) «Teaching and Learning in Small Rural Schools in Four European Countries. Introduction and Synthesis of Mixed-/Multi-Age Approaches». International Journal of Educational Research, 74, pp.97-103. DOI. 10.1016/j.ijer.2015.04.007.

Suedtirol Deutsche Schulamt. Reading Online. Recuperat de: http.//www.provinz.bz.it/schulamt/ [accés: 10/20/2017].

Tarozzi, M. (2008) Che cos'è la grounded theory. Roma, Carocci.

Thornberg, R. (2012) «Informed Grounded Theory». Scandinavian Journal of Educational Research, 56(3), pp. 243-259.

Tomazzolli, E.; Consalvo, G. (2018). «The Legacy of Maria Montessori on Multiage Classroom: Rethinking Small Rural Schools' Traditional Teaching-Learning Approach in Favor of Freedom of Choice». WERA Congress, Cape Town, South Africa.

Trentini, M.; Mirandola, G. (2016) Alta Scuola: storia di una utopia concreta. Antersass, Cierre.

Tuan, Y.F. (2003) II cosmo e il focolare. Milano, Eleuthera.

Westoby, P.; Shevellar, L. (Eds.) (2012) Learning and Mobilising for Community Development. A Radical Tradition of Community-Based Education and Training, Farnham, Ashgate.

White, S.; Corbett, M. (Eds.) (2014) Doing Educational Research in Rural Settings. Methodological issues, international perspectives and practical solutions. New York, Routledge.

Woods, M. (2011) Rural. Key Ideas in Geography. London, Taylor \& Francis.

Wylie, J. (2007) Landscape. London, Routledge. 


\section{Viatjar entre muntanyes, camps i petites illes: les escoles primàries rurals italianes són el motor del desenvolupament comunitari}

Resum: L'objectiu d'aquest treball és identificar els elements més característics de la relació existent entre les petites escoles rurals $i$ les comunitats on s'integren. Amb la mirada posada en els infants, aquesta investigació descriu la interacció entre nens, nenes, docents, famílies, autoritats educatives i polítics locals, ja siguin residents de llarga durada o famílies nouvingudes, sense perdre de vista l'entorn natural. S'ha adoptat un enfocament basat en una teoria constructivista i fonamentada. S'han visitat quinze petites escoles rurals italianes d'educació primària que inclouen como a mínim una aula multigrau. Els resultats van donar protagonisme a un element fonamental que transcendeix l'heterogeneïtat de les escoles visitades: la presència d'agents comunitaris molt motivats (docents, famílies, líders educatius, polítics i agents locals) que veuen l'escola com un motor per al desenvolupament de la comunitat. Entre aquests agents comunitaris s'ha posat especial atenció als docents durant l'anàlisi: el paper del mestre com a intel.lectual orgànic és el concepte que s'utilitza per definir el docent que viu la pròpia comunitat, però que té present la recerca històrica, literària, natural, musical i tot l'entorn.

Paraules clau: Relació escola-comunitat, escola rural, comunitat rural, aula multigrau, paper docent, política educativa.

\section{Viajar entre montañas, campos e islas pequeñas : las escuelas primarias rurales ita- lianas son el motor del desarrollo comunitario}

Resumen: El objetivo de este trabajo es identificar los elementos más característicos de la relación entre las pequeñas escuelas rurales y las comunidades en las que están integradas. Con la mirada centrada en los niños, esta investigación describe la interacción entre alumnos, docentes, familias, autoridades educativas y políticos locales, ya sea de familias residentes desde hace tiempo o bien de familias recién llegadas, sin perder de vista el entorno natural. Se ha adoptado un enfoque de teoría fundamentada constructivista y fundamentada. Se han visitado quince pequeñas escuelas rurales de educación primaria italianas que incluyen al menos una clase multigrado. Los resultados ponen de manifiesto un elemento fundamental que trasciende a la heterogeneidad de las escuelas visitadas: la presencia de agentes comunitarios altamente motivados (docentes, familias, líderes educativos, políticos y agentes locales) que ven la escuela como un motor para el desarrollo comunitario. Entre estos agentes de la comunidad se ha puesto especial atención a los docentes durante el análisis: el papel del maestro como intelectual orgánico es el concepto utilizado para definir al docente que vive en la comunidad, pero teniendo en cuenta la investigación histórica, literaria, natural, musical y todo el entorno

Palabras clave: Relación escuela-comunidad, escuela rural, comunidad rural, clase multigrado, rol docente, política educativa.

\section{Traveling around mountain areas, countryside and small islands: Italian rural pri- mary schools are the driving force for community development}

Abstract: The aim of this work is to identify the essential properties of the relationship between small rural schools and the communities in which they are located. Focussing on the children, this research describes the interaction between children, teachers, parents, local educational and political authorities, long-term residents and newcomers, as well as the natural environment. A constructivist and grounded theory approach was applied. Fifteen small Italian rural primary schools that include at least one multi-age classroom were visited. The outcomes reveal a fundamental element that stands out from the heterogeneity of the schools: the presence of highly motivated community agents (teachers, parents, educational leaders, politicians, and local agents) who see the school as an motor for community development. Among these community agents particular attention was paid to teachers during the analysis: the role of the teacher as organic intellectual is the concept used to define the teacher who lives in the community, but gives importance to historical, literary, natural, and musical research as well as the entire environment.

Keywords: School-community relationship, rural school, rural community, multiage classroom, teacher's role, educational policy. 